Haben Sie Fragen zur Abrechnung oder zur wirtschaftlichen Praxisführung? Als Leser der MMW können Sie sich an unseren Experten wenden: Helmut Walbert, Facharzt für Allgemeinmedizin und Betriebswirt aus Würzburg.

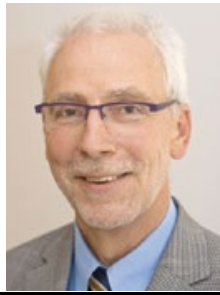

Helmut Walbert Allgemeinarzt, Medizinjournalist und Betriebswirt Medizin

\title{
GOÅ-Abrechnung
}

\section{Patient will Spritzen und Kanülen nicht zahlen}

\section{$?$ Dr. J. S., Allgemeinarzt, Bayern: Ein Patient weigert sich, im Rahmen einer Individuellen Gesundheitsleis- tung (IGeL) angefallene Sachkosten für Spritzen und Kanülen zu bezahlen - das hätte er bisher noch nie ge- musst. Hat er etwa recht?}

I Antwort: Auch IGeL unterliegen den Vorschriften der GOÄ. Die Erstattung von Sachkosten ist in $\$ 10$ geregelt. Hier ist im Absatz 1 festgehalten, dass Kosten für Arzneimittel, Verbandmittel und sonstigen Materialien, die der Patient zur weiteren Verwendung behält

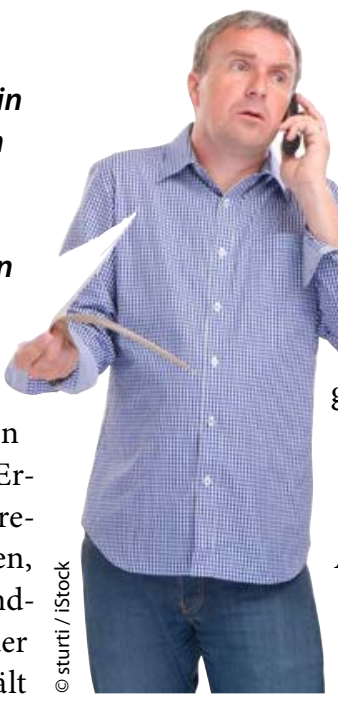

Manchem ist die Arztrechnung nur eine Diskussionsgrundlage.

oder die mit einer einmaligen Anwendung verbraucht sind, grundsätzlich abgerechnet werden können. Im Absatz 2 werden dann allerdings $\mathrm{Ma}$ terialien aufgezählt, die davon ausge- schlossen sind. Hierzu zählen Kleinmaterialien wie Zellstoff, Mulltupfer, Schnellverbandmaterial, Verbandspray, Holzspatel und Holzstäbchen, aber auch mehrere Einmalartikel - darunter auch Einmalspritzen und Einmalkanülen. Damit ist der Sachverhalt eindeutig: Der Patient muss nicht zahlen.

Dennoch muss Ihre Vorgehensweise grundsätzlich positiv gesehen werden. In vielen Arztpraxen werden die Sachkosten gar nicht verrechnet. Je nach Leistungsstruktur führt dies zu einem durchaus spürbaren Honorar- bzw. Gewinnverlust.

\section{Heillmittel}

\section{Folgeverordnung ist Sache des Erstverordners}

? Dr. H. G., Allgemeinarzt, Bayern: Ein Patienten hatte nach einer $O P$ in einer Klinik eine Heilmittelverordnung (HMV) erhalten. Wir haben ihm später eine weitere HMV ausgestellt, als Erstverordnung. Der Physiotherapeut hätte aber lieber eine Folgeverordnung von uns. Ist das korrekt? therapeuten ist es eine Folge-HMV.
Damit ist der Korrekturwunsch korrekt. Würde der Patient mit Ihrer „Erst“-Verordnung zu einem anderen Physiotherapeuten gehen, würde der Sachverhalt einer Folge-HVM nicht auffallen.

Eigentlich sollte aus medizinischen Gründen stets der Erstverordner auch die Folgeverordnungen ausstellen. So kann der Verlauf besser beobachtet und der Fortschritt des Gesundungsprozesses besser beurteilt werden. Dies gilt im
Prinzip auch bei Erst-HVM von Facharzt-Kollegen. In Ihrem speziellen Fall sollte sich die Klinik bei der Entlassung des Patienten auf eine Empfehlung beschränken. Oft wird in Kliniken mit der Ausstellung von HMV wesentlich großzügiger umgegangen, weil die Wirtschaftlichkeitskontrolle fehlt. Eine Rückverweisung an den Erstaussteller entlastet darüber hinaus Ihr Heilmittelbudget. 